\title{
Blutdruckmessung bei Typ-2-Diabetikern
}

Die Blutdruckwerte, die bei Typ-2-

Diabetikern die Diagnose einer Hypertonie begründen und die als Zielwerte der Therapie dienen, stützen sich vornehmlich auf konventionelle Messungen in Klinik und Praxis. Blutdruckwerte aus Langzeitmessungen sind aber prognostisch zuverlässiger.

- Bei 550 Typ-2-Diabetikern wurde der Blutdruck konventionell und mit ambulanter Langzeitmessung bestimmt (ABDM). Als Ausschlusskriterien galten u. a. Alter $>80$ Jahre, Serumkreatinin $>$ $2,0 \mathrm{mg} / \mathrm{dl}, \mathrm{BMI}>40 \mathrm{~kg} / \mathrm{m}^{2}$ und schwere Begleiterkrankungen.

Der systolische Blutdruck bei ABDM korreliert besser mit den diabetischen Komplikationen - ausgenommen Retinopathie, Nephropathie und koronare Komplikationen - als bei Praxismessung. Der diastolische ABDM-Druck ist ein deutlich schlechterer Indikator und korreliert signifikant nur mit Mikroalbuminurie, Nephropathie und zerebralen Komplikationen. Insgesamt sind die Werte in der Nacht aussagekräftiger als die Tageswerte. Das Blutdruckverhältnis von Nacht zu Tag und der fehlende nächtliche Abfall (non-dipping)

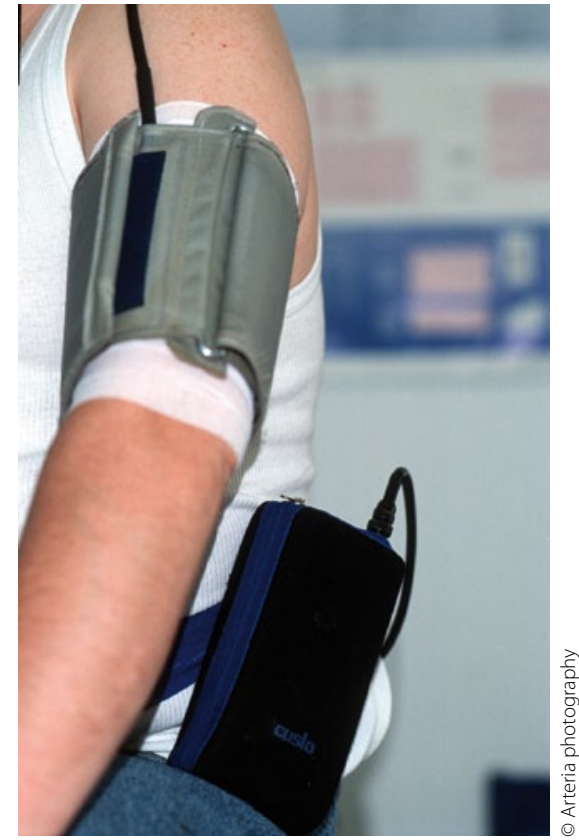

24h-RR-Messung: Eine zuverlässigere Basis für die Einstufung einer Hypertonie.

sind weniger eng mit allen diabetischen Komplikationen assoziiert als der 24-hMittelwert. Die Autoren leiten aus der Vielzahl der Einzelergebnisse für alle mikrovaskulären Komplikationen folgenden gemeinsamen Blutdruckgrenzwert ab: Tageswert 125/75 mmHg,
Nachtwert 110/65 mmHg und 24-Wert 120/75 mmHg.

\section{Kommentar}

Bisher resultierten alle Blutdruckgrenzwerte für diabetische Komplikationen aus Ergebnissen mit konventioneller Messung. Diese wurden dann - unter Abzug eines bestimmten Differenzbetrages - auf Werte für die Langzeitmessung übertragen. Die von den Autoren für die Langzeitmessung eruierten Werte bestimmen die Grenzen, ab der man bei Diabetikern mit typischen Komplikationen rechnen muss. Sie liegen niedriger als die seit einigen Jahren propagierten, neuerdings wieder in Frage gestellten Zielwerte unter antihypertensiver Therapie. (Die Deutsche Hochdruckliga etwa empfiehlt bei hypertonen Diabetikern einen Zielbereich von 130-140/80-85 $\mathrm{mmHg}$ ).

Grundsätzlich muss man bei allen Risikofaktoren berücksichtigen, dass Grenzwerte, ab denen Folgekrankheiten auftreten können, nicht identisch sein müssen mit den Zielwerten für die Therapie.

H. HOLZGREVE —

- C. R. L. Cardoso et al.

Thresholds of ambulatory blood pressure associated with chronic complications in type 2 diabetes. Am. J. Hypertens. 25 (2012) 82-88

\section{AchtUng:}

\section{Hier muss der Dummy durch eine Anzeige ersetzt werden !!}

